\title{
Ultradiscrete kinks with supersonic speed in a layered crystal with realistic potentials
}

\author{
J. F. R. Archilla, ${ }^{1, *}$ Yu. A. Kosevich, ${ }^{2, \dagger}$ N. Jiménez, ${ }^{3}$ V. J. Sánchez-Morcillo, ${ }^{3}$ and L. M. García-Raffi ${ }^{4}$ \\ ${ }^{1}$ Grupo de Física No Lineal, Universidad de Sevilla, ETSI Informática, Avda Reina Mercedes s/n, 41012 Seville, Spain \\ ${ }^{2}$ Semenov Institute of Chemical Physics, Russian Academy of Sciences. Kosygin street 4, 119991 Moscow, Russia \\ ${ }^{3}$ Instituto de Investigación para la Gestión, Integrada de las Zonas Costeras, Universidad Politécnica de Valencia, C/.Paranimfo 1, \\ 46730 Grao de Gandia, Spain \\ ${ }^{4}$ Instituto Universitario de Matemática Pura y Aplicada, Universidad Politécnica de Valencia, Camino de Vera s/n, 46022 Valencia, Spain
}

(Received 25 July 2014; published 13 February 2015)

\begin{abstract}
In this paper we develop a dynamical model of the propagating nonlinear localized excitations, supersonic kinks, in the cation layer in a silicate mica crystal. We start from purely electrostatic Coulomb interaction and add the Ziegler-Biersack-Littmark short-range repulsive potential and the periodic potential produced by other atoms of the lattice. The proposed approach allows the construction of supersonic kinks which can propagate in the lattice within a large range of energies and velocities. Due to the presence of the short-range repulsive component in the potential, the interparticle distances in the lattice kinks with high energy are limited by physically reasonable values. The introduction of the periodic lattice potential results in the important feature that the kinks propagate with the single velocity and single energy, which are independent on the excitation conditions. The unique average velocity of the supersonic kinks on the periodic substrate potential we relate with the kink amplitude of the relative particle displacements, which is determined by the interatomic distance corresponding to the minimum of the total, interparticle plus substrate, lattice potential. The found kinks are ultradiscrete and can be described with the "magic wave number" $q=2 \pi / 3 a$, which was previously revealed in the nonlinear sinusoidal waves and supersonic kinks in the Fermi-Pasta-Ulam lattice. The extreme discreteness of the observed supersonic kinks, with basically two particles moving at the same time, allows the detailed interpretation of their double-kink structure, which is not possible for the multikinks without an account for the lattice discreteness. Analytical calculations of the displacement patterns and energies of the supersonic kinks are confirmed by numerical simulations. The computed energy of the found supersonic kinks in the considered realistic lattice potential is in a good agreement with the experimental evidence for the transport of localized energetic excitations in silicate mica crystals between the points of ${ }^{40} \mathrm{~K}$ recoil and subsequent sputtering.
\end{abstract}

DOI: 10.1103/PhysRevE.91.022912

PACS number(s): 05.45.-a, 63.20.Pw, 63.20.Ry

\section{INTRODUCTION}

Many minerals are known by their capability of recording the tracks of charged particles and are often used as solid state nuclear track detectors (SSNTDs) [1,2]. Among them, silicate mica muscovite crystal has been relevant as it was the second material and the first natural one where the tracks from fission fragments were found [3]. Soon later fossil tracks were also found in silicate mica crystal [4]. This crystal seems to be one of the most sensitive of the natural SSNTDs [5]. Mica crystal has important technological applications due to its dielectric and heat-shielding properties. Due to its heat resistance, mica crystal can be used inside a nuclear reactor core for particle detection. It has also been used in geochronology and to probe the existence of dark matter [6], to find exotic nuclear reactions, decays of super heavy elements, and weakly interacting massive particles [1,2]. Silicate mica crystals are among the materials considered as engineered barriers for nuclear waste storage due to their high rate of reaction with heavy ions in low-temperature reconstructive transformations [7]. The latter are also important for the development of advanced structural ceramics [8]. The reconstructive transformations at low temperature were related with the existence of stationary

\footnotetext{
*archilla@us.es

${ }^{\dagger}$ Corresponding author: yukosevich@gmail.com
}

intrinsic localized vibrational modes (discrete breathers) in mica crystals $[9,10]$.

Tracks of positrons, muons, and other particles have been reported [11-14] in mica muscovite crystals. Some of these tracks were identified as being produced by positrons resulting from the $\beta+$ decay of ${ }^{40} \mathrm{~K}$. This isotope is relatively abundant in the minerals and can also experience the $\beta-$, electron capture and other kinds of decays $[15,16]$. Most of the tracks, however, cannot be explained as being produced by charged particles but could have been produced by some kind of propagating energetic vibrational excitation since the tracks are along the close-packed lines of the $K^{+}$hexagonal layer shown in Figs. 1 and 2. One interpretation of these tracks is that they are formed by the quasi-one-dimensional (1D) localized nonlinear excitations, sometimes called quodons [17], whose exact nature is still unknown.

A likely source for the vibrational energy required to initiate a quodon is the recoil energy of the ${ }^{40} \mathrm{~K}$ after $\beta$ decay, which can be up to $52 \mathrm{eV}[15,16]$. In mica muscovite crystals, there are about three decays per second and $\mathrm{cm}^{3}$, so after many years of the sensitive period, when track recording is possible, there are many possibilities to initiate a quodon. An experiment was done to shed some light into the relationship between tracks and quodons [18]. A mica specimen was irradiated by alpha particles, and the ejection of atoms was detected on the opposite side, along the close-packed lines. The ejected atoms could not been identified and sputtering energies are not known 


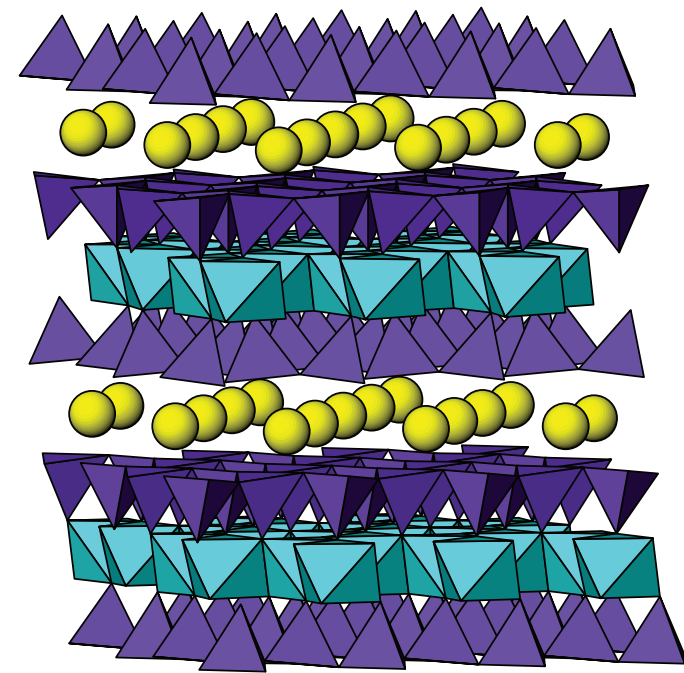

FIG. 1. (Color online) Representation of the mica structure from the point of view that emphasizes the close-packed lines of the $K^{+}$ hexagonal layer represented by yellow balls. For a view from the top, see Fig. 11

exactly; however, from experimental and theoretical studies, they are known to be in the range of $4-8 \mathrm{eV}$ [19].

In an attempt to understand this phenomenon, numerical simulations were conducted in an idealized 1D model where the particles with a given high energy hit the boundary of the lattice [20]. This study reports the formation of breathers and kinks in the lattice bulk and the ejection of atoms at the opposite boundary of the sample. However, the model did not intend to use realistic values of the mica muscovite parameters.

A minimal model of the cation lattice with realistic parameters has been proposed recently [21,22]. Only $\mathrm{K}^{+}$ions were considered with the actual potassium mass and Coulomb interaction between them. The interaction with the rest of the lattice was implicitly considered as the force keeping the ions inside the crystal with the known interatomic distance in the muscovite. In those publications, it was reported the existence of supersonic kinks which were easily generated within a wide range of velocities and energies.

However, such model is too simplistic because for relatively high ion energies it results in unrealistically small distances between the ions, of tenths of angstrom. In this paper, we develop a comprehensive dynamical model of the propagating nonlinear localized lattice excitations, supersonic kinks, in which we use the Ziegler-Biersack-Littmark (ZBL) shortrange repulsive potential, introduced in particle bombardment

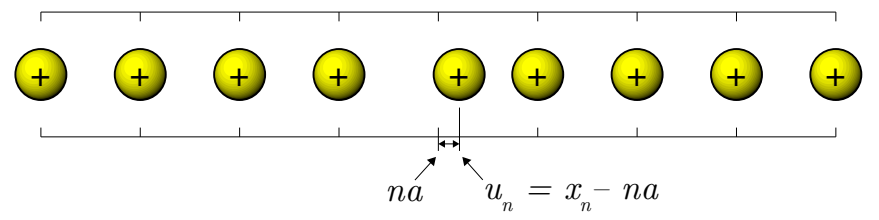

FIG. 2. (Color online) Representation of a close-packed line of $K^{+}$ions and the variables used. At equilibrium one has $x_{n}=n a$, and $u_{n}$ measures the displacement from equilibrium of the ion labeled $n$. In physical units $a=5.19 \AA$, and $a=1$ in scaled units. studies [23]. ZBL potential describes the Coulomb repulsion between nuclei, which is partially screened by the atom electrons and rapidly decays within few $\AA$. The results for the moving energy-carrying objects in such potential are similar to those obtained with pure Coulomb potential [21,22], namely, that supersonic kinks, traveling without attenuation for long distances, can be produced within a wide range of velocities and energies. The interparticle distances in the kinks in our dynamical model are limited by physically reasonable values. Note that our dynamical model allows for bond dissociation, which realizes one possible physical mechanism of the normal heat transport in low-dimensional systems [24]. From the other hand, the classical description of the supersonic kink formation and propagation is justified by the fact that ZBL potentials are known to provide very good agreement with the experiments on atom collisions in the context of radiation damage and ion track formation, which can be modeled using the methods of classical molecular dynamics [25-27].

The literature about kink propagation in lattices with different interparticle and on-site potentials is extensive. The most studied and generic model is the Frenkel-Kontorova (FK) model [28]; see reviews in Refs. [29-31]. However, most of the kinks considered in these publications are subsonic ones. Supersonic kinks in the systems with substrate have been found in models with anharmonic interparticle coupling [32-35]. They have the property that only a discrete set of velocities allows the propagation of the kinks without attenuation. They can be described as multikinks or lattice $N$ solitons depending on whether the description is done in terms of displacements, strains, or velocities.

The FK model has also been considered in layered materials, to model, for example, the in-plane dynamics of a few-layer graphene, in order to explain the results of molecular dynamics simulation of the cross-plane thermal conductance [36].

As a next step towards a more realistic description, we construct explicitly the interaction with the surrounding atoms, using standard empiric potentials introduced in molecular dynamics, which give rise to a periodic nonsinusoidal substrate potential. Supersonic lattice kinks, which are also called crowdions [32], were constructed in our dynamical model. They propagate in such potential with a velocity which is independent of the excitation conditions and is determined only by the lattice potential parameters. The kink (crowdion) with the unique supersonic velocity, propagating in our dynamical model of the cation layer on a substrate, has a double-kink structure. We relate the unique velocity of the supersonic kink on the periodic substrate potential with the kink amplitude of the relative particle displacements, which is determined by the interatomic distance corresponding to the minimum of the total, interparticle plus substrate, lattice potential.

The important characteristic, found in the kinks studied in the present work, is extreme discreteness of the kinks, with only one or two particles in motion at a given time. The extreme kink discreteness allows the complete understanding in physical terms of the mechanism that brings about the double-kink structure of the found kinks.

The computed energy of the found supersonic kinks in the considered realistic lattice potential is approximately $26 \mathrm{eV}$ : 
Such energy can be provided by the recoil of isotopes of potassium after radioactive decay, and it is larger than the sputtering energy. This value of the characteristic energy of the found supersonic kinks allows to assume that the tracks found in mica muscovite crystals can be related with the propagating lattice kinks (crowdions).

We describe the Hamiltonian systems, in which the supersonic kinks under certain conditions lose their energy by emitting phonons; see Sec. V. But nevertheless the considered systems continue to be the strongly underdamped systems. Therefore the known properties of the kinks (fronts) in dissipative overdamped systems [37,38], including discrete ones [39,40], cannot be applied directly to the considered ultradiscrete supersonic kinks. For instance, due to the kink discreteness on the atomic scale, the position of the kink (front) core cannot be unambiguously defined as the position of the maximum of the displacement derivative $\partial_{x} u_{x}$ [39] because the latter quantity is not well defined for the ultradiscrete kink. Therefore we can define and numerically measure only the average kink velocity and cannot measure the possible weak time-domain oscillations of the kink speed and snaking bifurcation diagram [39,40].

The paper is organized as follows. First, we review and extend the results obtained with only Coulomb interaction, using the sinusoidal wave form with the magic wave number $q=2 \pi / 3 a$ proposed for supersonic kinks in the FermiPasta-Ulam (FPU) chain [41,42]. The sinusoidal wave form is a good description for $\lambda \simeq 3 a$, where $\lambda$ is a characteristic wavelength of the sinusoidal wave form and $a$ is the lattice constant, but fails for $\lambda \simeq 2 a$, been replaced by an almost triangular wave form, corresponding to nearly hard-sphere collisions. Afterwards, we consider the effects of long-range interactions with several neighbors, and introduce a short-range nearest-neighbor ZBL potential. Thereafter, the substrate potential is constructed and the properties of the single-velocity lattice kinks in the cation layer on the substrate are analyzed with detail. Finally we provide a summary and discussion of all the main results of the paper.

\section{MODEL AND SINUSOIDAL KINK DESCRIPTION}

We consider as a starting point a 1D model for the dynamics of $\mathrm{K}^{+}$ions, given in dimensionless form by

$$
\ddot{u}_{n}=-\frac{1}{\left(1+u_{n+1}-u_{n}\right)^{2}}+\frac{1}{\left(1+u_{n}-u_{n-1}\right)^{2}},
$$

which describes a chain of ions coupled to their nearest neighbors by electrostatic potential. The variables $u_{n}$ represent the displacement of a particle in the chain with respect to its equilibrium position, normalized to the lattice constant. The values of the scaled units are the following: for lengths, the lattice constant, the equilibrium distance between $K^{+}$ ions, $u_{L}=a=5.19 \AA$; for masses, the mass of a $K^{+}$ion, $m_{K^{+}}=39.1 \mathrm{amu}$; for time, $\tau=\sqrt{m_{K} a^{3} / \mathrm{k}_{\mathrm{e}} \mathrm{e}^{2}} \simeq 0.2 \mathrm{ps}, \mathrm{k}_{\mathrm{e}}$ is the Coulomb constant, and $e$ is the elementary unit of charge. Other physical units in the system are velocity $u_{V}=$ $2.6 \mathrm{~km} / \mathrm{s}$, energy $u_{E}=2.77 \mathrm{eV}$, momentum $u_{P}=1.698 \times$ $10^{-22} \mathrm{~kg} \mathrm{~m} / \mathrm{s}$, and frequency $5 \mathrm{THz}$. The dimensionless speed of sound in this system is $c_{s}=\sqrt{2}$, or $3.7 \mathrm{~km} / \mathrm{s}$ in physical units.
For small amplitudes, the potentials in Eq. (1) can be expanded in series using that $1 /(1+y)^{2} \simeq 1-2 y+3 y^{2}-$ $4 y^{3} \ldots$ Retaining cubic and smaller terms, we obtain

$$
\begin{aligned}
\ddot{u}_{n}= & c_{s}^{2}\left[\left(u_{n+1}+u_{n-1}-2 u_{n}\right)\right. \\
& -3 / 2\left(u_{n+1}-u_{n}\right)^{2}+3 / 2\left(u_{n}-u_{n-1}\right)^{2} \\
& \left.+2\left(u_{n+1}-u_{n}\right)^{3}-2\left(u_{n}-u_{n-1}\right)^{3}+\cdots\right],
\end{aligned}
$$

which are the $\alpha-\beta$ FPU equations of motion. We would like to emphasize that the $\alpha-\beta$ FPU equation (2) describes the Coulomb lattice (1) only in the small and intermediateamplitude limit and is not applicable to this lattice in the large-amplitude limit; see Fig. 4 and the subsection dedicated to the triangular wave form below.

Without nonlinear terms, Eq. (2) is reduced to the discrete linear wave equation:

$$
\ddot{u}_{n}=c_{s}^{2}\left(u_{n+1}+u_{n-1}-2 u_{n}\right),
$$

where $c_{s}$, the speed of sound, is the long wavelength phonon velocity. It is also both the maximum phase and group velocity. Note that in our scaling $c_{s}=\sqrt{2}$.

Introducing a new variable, the deformation from the equilibrium position or strain $v_{n}=u_{n}-u_{n-1}$, the equations above can be written as

$$
\ddot{v}_{n}=2 F_{n}-F_{n+1}-F_{n-1}, \quad \text { with } \quad F_{n}=\frac{1}{\left(1+v_{n}\right)^{2}},
$$

where $v_{n}=0$ corresponds to the unperturbed lattice. The boundary conditions assume fixed particles at the ends of the lattice. Kinks are produced numerically by applying at the chain boundary a half-wave perturbation, which is a sinusoidal displacement during half of a period, starting and finishing at the equilibrium position $[21,42]$. In order to describe a kink traveling to the right, the following ansatz was introduced in Refs. [41,42]:

$$
v_{n}=-\frac{A}{2}[1+\cos (q n-\omega t)] \quad \text { if } \quad-\pi \leqslant q n-\omega t<\pi
$$

and $v_{n}=0$ otherwise, where $A$ is the kink amplitude of the relative particle displacements. The bonds are always compressed so $v_{n}$ is negative with a minimum value of $-A$, corresponding to the maximum compression of the bond. For analogy, we use the usual wave terminology, so $\phi_{n}=\omega t-q n$ is the phase; $q$ is the effective wave number; $\omega$ is the angular frequency; $T$ is the period; $\lambda=2 \pi / q$ is the wavelength. The average velocity of the kink is defined as $V=\omega / q$ : It is determined by the time the kink needs to travel a lattice site. Numerically it is measured by tracing the states of maximum lattice compression.

The sinusoidal wave form with the magic wave number $q=2 \pi / 3$ is an exact solution for the nonlinear sinusoidal waves in the FPU chain [41-43], which is characterized by the translational-invariant interparticle potential with cubic and quartic anharmonicity. This potential is a good approximation for many realistic potentials, including the Coulomb one, for the intermediate amplitudes. Such wave form provides a very useful framework for understanding the relative phases of the moving particles and the behavior of the kink. 

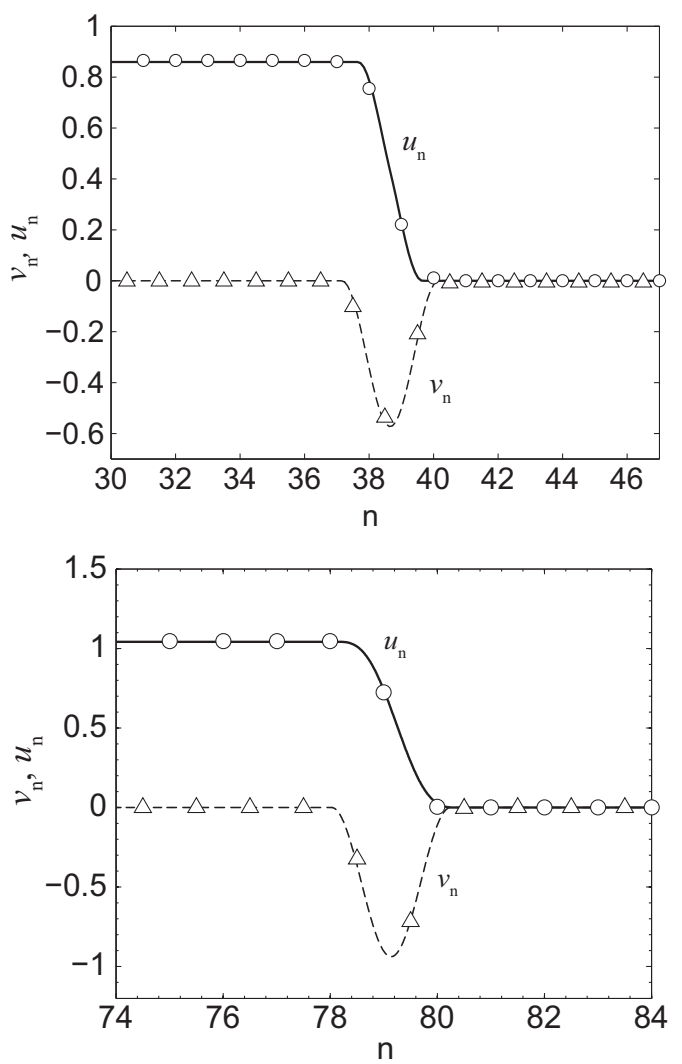

FIG. 3. Profile for the two kinks coordinates $u_{n}$ and strains $v_{n}=$ $u_{n}-u_{n-1}$. Circles and triangles are numerical results, and the continuous lines are obtained from Eq. (5) with magic wave number $q \simeq$ $2 \pi / 3$ (top), when basically two particles are moving at a given time, and with $q \simeq \pi$ (bottom) for the amplitude $A$ close to 1 , when basically one particle is moving at a given time. Scaled units are equal to the lattice unit.

Figure 3 (top) compares the result of the numerical simulation for displacements and strains, with the corresponding analytical expressions derived from Eq. (5), for the intermediate value of the amplitude $A=0.55$ and magic wave number $q \simeq 2 \pi / 3$. The analytical expression fits very well the numerical data. Figure 3 (bottom) pictures a kink with wave number $q=\pi$. There are not enough particles to compare with the analytical form, but the main properties of the latter are valid.

For the dimensionless wave number $q=2 \pi / \lambda$, with $\lambda$ an integer, Eq. (5) represents a solution where basically $\lambda$ bonds and $\lambda-1$ particles (the kink core) are in motion, while the others remain at rest.

We will use the term active to describe related states of the different magnitudes. The active particles or coordinates at a given time or time interval (or phase or phase interval) are those for which $u_{n}$ is changing, the active bonds are those for which $v_{n} \neq 0$; i.e., they are the compressed ones. For a particle, the time interval is active when it is moving, and for a bond, when it is compressed.

If the dimensionless wavelength $\lambda$ is between two integers $m_{1}$ and $m_{2}$, the number of active bonds oscillates between $m_{1}$ and $m_{2}$ and the number of active oscillating particles is between $m_{1}-1$ and $m_{2}-1$.

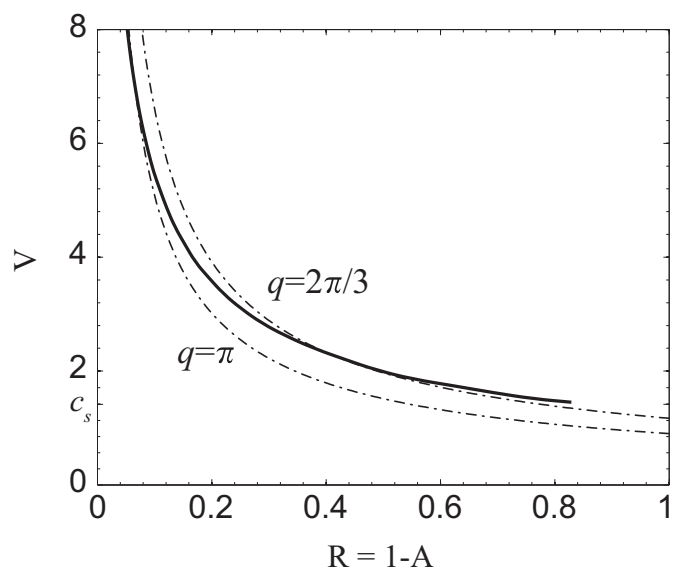

FIG. 4. Kink velocity versus minimal interparticle distance $R=$ $1-A$, calculated numerically (thick line) and analytically from Eq. (6) with wave number $q=2 \pi / 3$ (upper dotted-dashed line) and $q=\pi$ (lower dotted-dashed line). Scaled units are the lattice constant for distances and $2.6 \mathrm{~km} / \mathrm{s}$ for velocities.

Of particular interest in this work will be $\lambda=3$, with $q=$ $2 \pi / 3$, which is called the magic wave number [42], and $\lambda=2$, with $q=\pi$, which will be referred to as the $\pi$ mode. These two values are extreme cases of localization, with $q=\pi$ being the limit when only one particle is moving at a given time.

\section{A. Rotating wave approximation}

The average velocity of the kink can be analytically obtained with the use of the rotating wave approximation (RWA) as derived in Ref. [21]:

$$
V=\frac{\omega}{q}=\frac{1}{(1-A)^{3 / 4}} c_{s} \frac{\sin (q / 2)}{(q / 2)} .
$$

Kinks are therefore supersonic. In the small amplitude $(A \rightarrow 0)$ and long wavelength $(q \rightarrow 0)$ limit, $V$ tends to the sound speed $c_{s}$.

In Ref. [21] it was shown that the wave form with the magic wave number demonstrates a good agreement between the ansatz and simulations. However, this agreement fails at the kink amplitudes $A$ approaching unity, when the minimal interparticle distance in the kink (in lattice units) $R=1-A$ diminishes and the $q=\pi$ brings about a much better fit as can be seen in Fig. 4. The conclusion is that the magic wave number is a suitable approximation for an intermediate range of amplitudes in the Coulomb lattice, in contrast to the FPU chain where it is valid for all amplitudes [41-43], and that $q$ actually changes continuously with the amplitude in the Coulomb lattice. The complementary approach is to use Eq. (6) to find the values of the wave number $q$, with respect to the amplitude $A$ or the velocity $V$. This will be shown in Fig. 9, where it can be appreciated that the wave form with the magic wave number is a proper solution in the Coulomb lattice for the intermediate values of the kink velocity. 


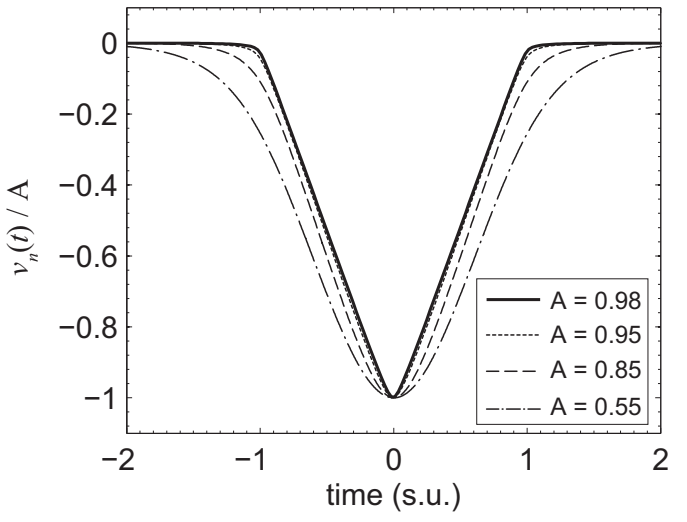

FIG. 5. Temporal variation of the strain $v_{n}=u_{n}-u_{n-1}$ for different amplitudes. As the amplitude $A$ increases, the shape becomes almost triangular, corresponding to almost hard-spheres collisions. The amplitude $A$ is given in lattice units and the scaled time unit is 2 ps.

\section{B. Triangular waveform}

For the higher amplitudes, with $A$ close to 1 , the wave form deviates from the sinusoidal one given by Eq. (5) and approaches instead the triangular wave form, which is shown in Fig. 5. The almost straight lines in such wave form mean that the velocity is almost constant except during a very short interaction time. The system behavior is very similar to that of the hard-spheres model.

This wave form is also another way of looking at the mode $q=\pi$. In this mode only one particle is moving at a given time, which means that there are no forces on the particle acting from its neighbors. Strictly speaking, the mode $q=\pi$ and an exact triangular wave form are unattainable because of the electrostatic Coulomb forces acting on the particles. However, if the energy of the particle is large with respect to the change in the potential during a large part of the path between collisions, the particle will move almost free during most of the time between collisions. The triangular wave form has been described as the high-energy limit for the Lennard-Jones interatomic potential [44] and has been observed experimentally in a system of repelling magnets [45]. It is worth mentioning that the triangular wave form can also be related with strongly stretched bonds in the high-energy limit in the potentials allowing for bond dissociation, like the Lennard-Jones potential. The strongly stretched bonds result in finite (normal) thermal conductivity in 1D systems with such interatomic potentials [24].

\section{Analytical results}

\section{Sinusoidal waveform and mode with $q=2 \pi / 3$}

Some analytical results can be obtained in this model; see also Ref. [42]. Let us consider the wave number $q=2 \pi / 3$ and choose $t=0$ for the time for which $\phi_{n}=q n-\omega t=0$ at $n=0$ after a change of the origin of $n$ and $t$, but keep the notation $n$ for generality. If we consider the time interval $\Delta t:-T / 6 \leqslant t<T / 6$, there are three active strains: $v_{n+1}, v_{n}$, and $v_{n-1}$. At the end of the interval, that is at $t=T / 6, v_{n-1}$ becomes zero and $v_{n+2}$ starts being perturbed, so all the indexes $n$ of the particles will change in a unity.

During $\Delta t, u_{n^{\prime}}=0$ for $n^{\prime} \geqslant n+1, u_{n}$ and $u_{n+1}$ are changing but $u_{n-1}$ has already attained the value $3 A / 2$, its final constant value as can be checked by direct sum. Also, $u_{n^{\prime}}=3 A / 2$ for $n^{\prime}<n-1$, their final value after the kink has passed over them as seen in Fig. 3. The active coordinates are therefore $u_{n}=-v_{n+1}$ and $u_{n-1}=-v_{n}+v_{n+1}$. After some algebra we get

$$
\begin{aligned}
u_{n} & =\frac{A}{2}+\frac{A}{2} \cos \left(\phi_{n}+2 \pi / 3\right), \\
u_{n-1} & =A-\frac{A}{2} \cos \left(\phi_{n}-2 \pi / 3\right) .
\end{aligned}
$$

We can obtain immediately the kink kinetic energy as $K=$ $\frac{1}{2} \dot{u}_{n}^{2}+\frac{1}{2} \dot{u}_{n-1}^{2}$, which results in

$$
K=\frac{\omega^{2} A^{2}}{8}\left[1+\frac{1}{2} \cos \left(2 \phi_{n}\right)\right]
$$

with maximum value

$$
K_{M}=\frac{\pi^{2}}{12} V^{2} A^{2} .
$$

The potential energy, with respect to the equilibrium state, is given by

$$
U=\frac{1}{1+v_{n-1}}+\frac{1}{1+v_{n}}+\frac{1}{1+v_{n+1}}-3 .
$$

By substitution, it can be seen that the maximum potential energy corresponds to the bond $n$ at its maximum compression, i.e., with $\phi_{n}=0$, while the bonds $n-1, n+1$ have a phase difference of $\pm 2 \pi / 3$ with a strain $u_{n-1, n+1}=-A / 2(1+$ $\cos ( \pm 2 \pi / 3)=-A / 4$. Therefore the maximum energy becomes

$$
U_{M}=\frac{1}{1-A}+\frac{2}{1-A / 4}-3 .
$$

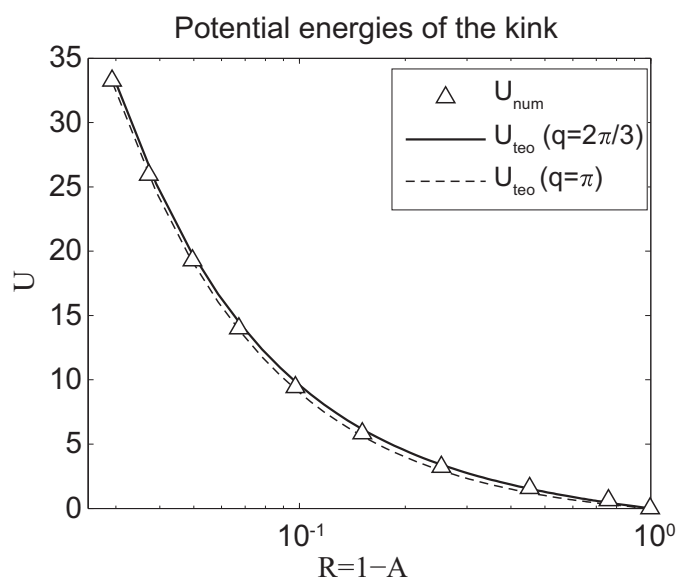

FIG. 6. Maximum potential energies of the kinks in the Coulomb potential versus minimal interparticle distance in lattice units $R=$ $1-A$ (for amplitude $A$ close to 1 ). The analytical results are very similar for the wave forms with $q=\pi$ and $q=2 \pi / 3$, as the maximum potential energy of the kink depends mainly on the minimal separation between particles $R$. The values of the dimension units are the lattice unit and $2.77 \mathrm{eV}$, respectively. 
There is also a minimum potential energy which corresponds to the limit, in which only two bonds are different from zero with phases $\pm \pi / 3$ and strain $v_{n}=-3 A / 4$. The minimum energy becomes

$$
U_{m}=\frac{2 A}{1-3 A / 4}-2
$$

The kink has always some compression energy $U_{m}$ above the ground state.

\section{Sinusoidal waveform and mode with $q=\pi$}

The properties of sinusoidal kinks with mode $q=\pi$ are easy to obtain as there are only two active strain variables and one coordinate, which during the interval $\Delta_{\pi} t, 0 \leqslant t<T / 2$, are

$$
\begin{aligned}
v_{n} & =-\frac{A}{2}[1+\cos (q n-\omega t)], \\
v_{n+1} & =-\frac{A}{2}[1+\cos (q[n+1]-\omega t)] \\
& =-\frac{A}{2}[1-\cos (q n-\omega t)], \\
u_{n} & =-v_{n+1} ; \quad \dot{u}_{n}=\frac{A}{2} \omega \sin (q n-\omega t) .
\end{aligned}
$$

The kinetic and potential energies, $K=\frac{1}{2} \dot{u}_{n}^{2}$ and $U=\frac{1}{1+v_{n}}+$ $\frac{1}{1+v_{n+1}}-2=\frac{1}{1+v_{n}}+\frac{1}{1-v_{n}}-2$, can be obtained. The maximum kinetic, maximum, and minimum potential energies are given by

$K_{M}=\frac{\pi^{2}}{8} A^{2} V^{2}, \quad U_{M}=\frac{1}{1-A}-1, \quad U_{m}=\frac{2}{1-A / 2}-2$.

\section{Triangular wave form and mode with $q=\pi$}

The potential energy fits very well the numerical values, unlike the kinetic energy, as can be seen in Figs. 6 and 7. This is because the $\pi$ kink is better described by the triangular form for large energies. Let us suppose that $t=0$ is the time for which $v_{n}=-A$, as it takes half a period to change from $-A$ to 0 , then $V T / 2=2$. Therefore, for the interval $0 \leqslant t<T / 2$ the active variables are

$$
\begin{aligned}
& v_{n}=-A+A V t, \quad v_{n+1}=-A V t, \\
& u_{n}=-v_{n+1}=A V t, \quad \dot{u}_{n}=A V .
\end{aligned}
$$

The kinetic energy is therefore a constant:

$$
K=K_{M}=\frac{1}{2} A^{2} V^{2} .
$$

In the numerical simulations, there are short time intervals when $K$ changes, separated by a larger interval when $K$ is almost constant.

The maximum and minimum potential energies are identical to that of the $q=\pi$ sinusoidal wave form given by Eq. (14).

\section{INTERACTION WITH SEVERAL NEIGHBORS}

All the previous results apply to the case of nearest-neighbor coupling. We have also checked that the kinks still exist

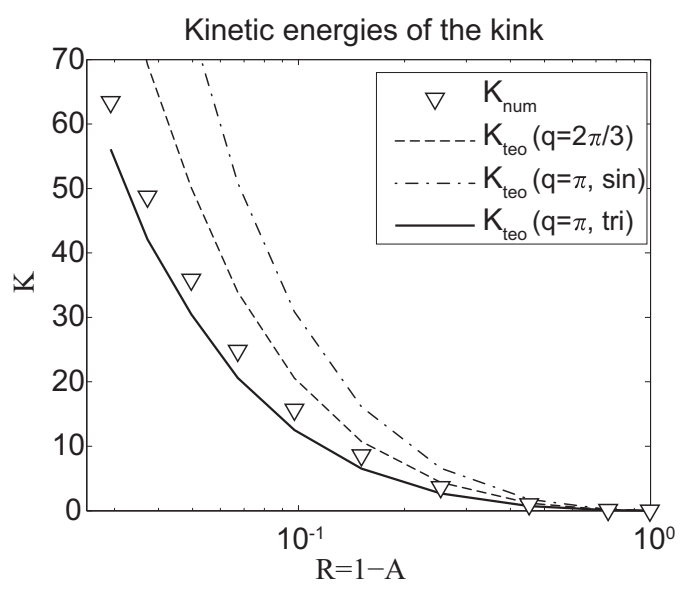

FIG. 7. Maximum kinetic energies of the kinks in the Coulomb potential versus minimal interparticle distance in lattice units $R=$ $1-A$ (for amplitude $A$ close to 1 ). It can be seen that the kink with wave number $\pi$ is better described by a triangular wave form than by a sinusoidal one. The values of the dimension units are the lattice unit and $2.77 \mathrm{eV}$, respectively.

and propagate supersonically when the Coulomb interaction extends beyond the nearest neighbors. The kinks have similar velocity and wave number if we take as reference a normalized velocity $V / c_{p}$, where $c_{p}=c_{s}\left(\sum_{l=1}^{p} 1 / l\right)^{1 / 2}$ is the sound speed in the Coulomb chain with interactions between $p$ nearest neighbors. Figure 8 shows the dependence of the relative velocity $V / c_{p}$ on the minimum interparticle distance, and Fig. 9 shows the dependence of the best fit for the wave number

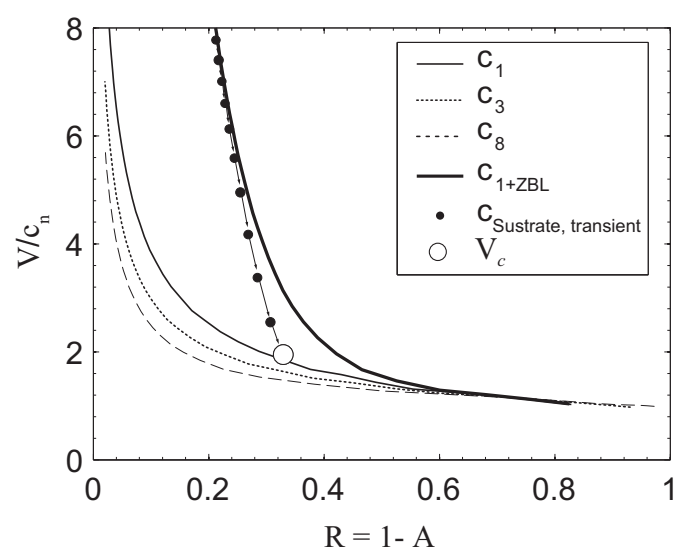

FIG. 8. Kink velocities as a function of minimal interatomic distance in lattice units $R=1-A$ ( $A$ is kink's amplitude) for several potentials. Notations in the legend: $C_{i}, i=1,3,8$, refers to the Coulomb interaction between the first $i$ neighbors. Velocities are normalized to the sound speed $c_{p}$ for a system with interaction between $p$ neighbors, except for the system with a substrate, which is normalized to $c_{1}=c_{s}$. It can be seen that the increase of the number of interacting neighbors slows the normalized velocity $V / c_{p}$ of the kink (but not its absolute velocity). Also, it can be seen that in the system with a substrate the normalized velocity $V / c_{p}$ deviates from the curve $c_{1+\mathrm{ZBL}}$ to some specific velocity $V_{c}$ on the $c_{1}$ curve. The values of the sound velocities are $c_{1}=c_{s}=3.7 \mathrm{~km} / \mathrm{s}, c_{3}=5.0 \mathrm{~km} / \mathrm{s}$, and $c_{8}=6.1 \mathrm{~km} / \mathrm{s}$. 


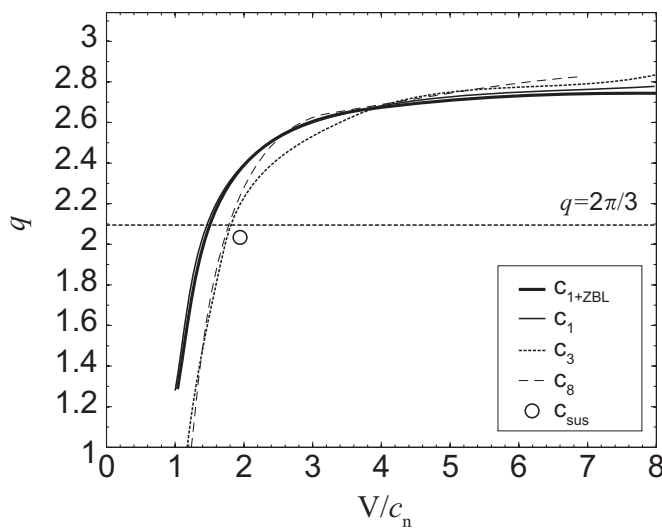

FIG. 9. Best fit for the wave number $q$ of the kinks as a function of the velocities normalized to the sound speed. Notations in the legend: $C_{i}$ is the Coulomb interaction between the first $i$ neighbors. Note that the magic wave number $q=2 \pi / 3$ is also chosen in the system with a substrate. The values of the sound velocities are $c_{1}=c_{s}=3.7 \mathrm{~km} / \mathrm{s}$, $c_{3}=5.0 \mathrm{~km} / \mathrm{s}$, and $c_{8}=6.1 \mathrm{~km} / \mathrm{s}$.

as a function of the relative velocity, emphasizing the essential identity of the phenomenon. Other aspects of these figures will be commented below. For clarity, only the cases of three and eight neighbors are represented although up to 30 neighbors have been tested.

It is worth commenting the problem that arises when the interaction with an infinite number of neighbors is taken into account. If the pair potential depends on the interparticle distance $|r|$ as $1 /|r|^{s}$, the long-wave phonon velocity is finite for $s>1$, but it diverges with the number of particles $N$ as $v_{\mathrm{ph}} \propto \sqrt{\ln (N / 2)}$ for the unscreened Coulomb potential $(s=1)$. However, this divergence occurs only in the electrostatic limit when the electromagnetic wave retardation is neglected. With an account of the retardation, the long-wave group velocity tends to the speed of light in vacuum. If the particles are in a material medium, there is a rearrangement of the electron density that can be described as a screening of the Coulomb interaction with some characteristic length $l_{\text {scr. }}$. The screening brings about a finite long-wave phonon velocity $v_{\mathrm{ph}} \propto \sqrt{\ln \left(l_{\mathrm{scr}} / a\right)}$, where $a$ is the lattice constant. As has been mentioned, we do not study in depth this problem here and have considered only a few neighbors for simplicity.

\section{KINKS WITH SHORT-RANGE ZBL POTENTIAL}

The minimal interatomic distance in lattice units $R=$ $1-A$, obtained for fast large-amplitude kinks, is clearly impossible in realistic systems. At short distances shortrange forces appear, which are produced by the overlapping electronic shells of the two close atoms. A large number of different repulsive potentials and screening functions have been proposed over the years, some determined semiempirically, others from theoretical calculations. A much used repulsive potential is the one given by Ziegler, Biersack, and Littmark, the so-called ZBL repulsive potential. It has been constructed by fitting a universal screening function to theoretically obtained potentials calculated for a large variety of atom pairs [23]. The ZBL potential has the form

$$
U_{\mathrm{ZBL}}(r)=\mathrm{k}_{\mathrm{e}} \frac{Z_{1} Z_{2} \mathrm{e}^{2}}{r} \phi\left(\frac{r}{\rho}\right),
$$

with $\mathrm{k}_{\mathrm{e}}$ being the Coulomb constant, $Z_{1}$ and $Z_{2}$ are the atomic numbers of the involved atoms, and $r$ the distance between them, $\phi(x)$ is the universal screening function:

$$
\begin{aligned}
\phi(x)= & 0.1818 \exp (-3.2 x)+0.5099 \exp (-0.9423 x) \\
& +0.2802 \exp (-0.4029 x) \\
& +0.02817 \exp (-0.2016 x) .
\end{aligned}
$$

The screening length is $\rho=0.8854 a_{0} /\left(Z_{1}^{0.23}+Z_{2}^{0.23}\right), a_{0}=$ $0.529 \AA$ being the Bohr radius. The ZBL potential describes well the interaction between neutral atoms. In the case of ions considered here, the Coulomb potential must also be added, accounting for the repulsion between the ions. The introduction of the $\mathrm{ZBL}$ potential restricts the interatomic distances to the realistic values. The four terms in the ZBL potential are important for different range of energies, but for $K^{+}$ions, with energies up to $200 \mathrm{KeV}$, which is much larger that the ones considered here, the interaction potential can be represented by a single term, which together with the Coulomb ionic part takes the form

$$
U(r)=\frac{1}{r}+\frac{\alpha}{r} \exp \left(-\frac{r}{\rho}\right)
$$

with $\alpha=184.1$ and $\rho=0.0569$ in dimensionless units, which in physical units correspond to $2650.6 \mathrm{eV} \AA$ and 0.29529 $\AA$, respectively. Figure 10 represents ZBL and Coulomb potentials, with their sum and other details to be commented on later. Note that around $r \simeq 0.4$, the combined potential $U(r)$ differs considerably from the Coulomb potential.

The dynamical equations become

$$
\ddot{u}_{n}=-G_{n+1}+G_{n}-F_{n+1}+F_{n},
$$

with $F_{n}$ given by Eq. (4) and $G_{n}$ given by

$$
G_{n}=\frac{\alpha}{1+v_{n}} \exp \left(-\frac{1+v_{n}}{\rho}\right)\left(\frac{1}{1+v_{n}}+\frac{1}{\rho}\right) .
$$

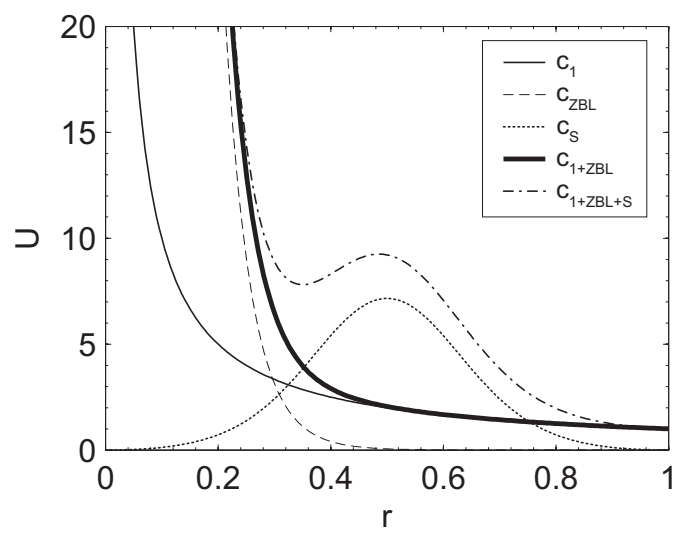

FIG. 10. Interaction potentials $U(r)$ in dimensionless units. Coulomb $\left(c_{1}\right)$; ZBL ( $\left.c_{\mathrm{ZBL}}\right)$; Coulomb +ZBL $\left(c_{1+\mathrm{ZBL}}\right)$; substrate potential $\left(c_{\mathrm{S}}\right)$ and the sum of the Coulomb, ZBL and substrate potentials $\left(c_{1+\mathrm{ZBL}+\mathrm{S}}\right)$. The scaled units are $2.77 \mathrm{eV}$ and the lattice unit $a=5.19 \AA$ for $U$ and $r$, respectively. 
When the joint effect of both the screened Coulomb (ZBL) and bare Coulomb potentials is considered, i.e., Eq. (19), numerical simulations show that the behavior of the kinks is not much different from that observed in the bare Coulomb case, discussed in the preceding sections. Supersonic kinks propagate equally well, changing from the magic wave number $q=2 \pi / 3$ to the proximity of $q=\pi$ and from the sinusoidal to nearly triangular wave form when the amplitude $A$ increases. Figures 8 and 9 also show the characteristic curves $V=V(A)$ and $q=q(A)$. The sound velocity does not change since the ZBL potential is felt only for very large perturbations. The RWA cannot be obtained analytically but the numerical RWA fits very well the results of the simulations.

\section{THE EFFECT OF THE SUBSTRATE POTENTIAL: LATTICE KINKS OR CROWDIONS}

In the preceding sections, the interaction with the other atoms in the crystal was taken into account only implicitly, since the only effect of the surrounding atoms was to fix the equilibrium lattice period and to confine the particles within the crystal. To better model the properties of the kinks in a crystal like muscovite, we take into account explicitly the interaction with the surrounding atoms in a simplified mica geometry. The $\mathrm{K}^{+}$ions occupy the nodes of the hexagonal lattice with a lattice unit 5.19 ̊. There are no other atoms in the $\mathrm{K}^{+}$plane; therefore we need to consider more atoms above and below. We consider two planes above and two symmetric below. The closest plane, at the distance of $1.6795 \AA$, is occupied with oxygen ions with charge -2 ; their projections on the $\mathrm{K}^{+}$plane lie in the middle of the two nearest-neighbor $\mathrm{K}^{+}$ions. The other layers, at the distance of $2.2227 \AA$ from the $\mathrm{K}^{+}$plane, are occupied by silicon ions. They are in the centers of tetrahedra whose three horizontal vertices are occupied by the oxygen ions. See Fig. 11 for a sketch. The $\mathrm{Si}$ sites are occupied by $\mathrm{Si}^{+4}$ and $\mathrm{Al}^{+3}$ ions in the proportion of $3: 1$, giving an average charge of +3.75 , but we assign them a smaller charge +2.75 to take into account other atoms in successive layers, particularly the oxygen ions at the top of the tetrahedra, and to achieve charge neutrality. We suppose that all the atoms are in fixed positions except the moving $\mathrm{K}^{+}$ions in a row in the [100] direction. This is justified by the supersonic speed of the kinks, that we are interested in, and due to the weak interaction between the $\mathrm{Si}$ and $\mathrm{Al}$ ions compared to the $\mathrm{ZBL}$ interaction between potassium ions.

The interaction between the $\mathrm{K}^{+}$ions, that are in the central $X$ axis, are not considered in constructing the substrate potential as it is taken into account explicitly. The lattice is extended in the $(001) \mathrm{K}^{+}$plane until the convergency of the potential is achieved.

Specifically the potentials used are the electrostatic interactions and the Born-Mayer potentials of the form $V=$ $A \exp \left(-r / r_{g}\right)$, given in Ref. [46]. The value $r_{g}=0.29 \AA$ is for all the interactions, and the pre-exponential constants in $\mathrm{eV}$ are $A_{\mathrm{KO}}=3800.125, A_{\mathrm{KSi}}=2762.5, A_{\mathrm{OO}}=453.375, A_{0 \mathrm{Si}}=$ 1851.25 , and $A_{\mathrm{SiSi}}=1173.125$. For the $\mathrm{K}^{+}-\mathrm{K}^{+}$interaction, we use the ZBL potential described above.

We obtain a substrate potential in scaled units $\left(u_{E} \simeq\right.$ $2.77 \mathrm{eV})$, which can be described with very good approxi-

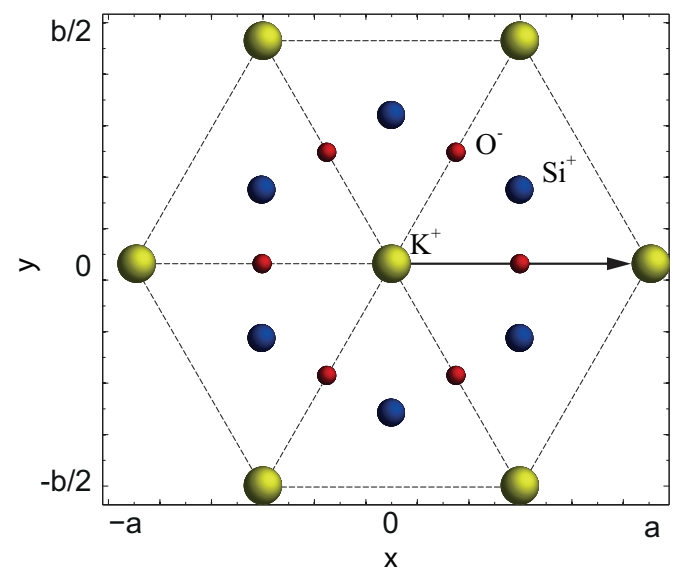

FIG. 11. (Color online) Projection on the (001) plane of the ions used in the calculation of the substrate potential. Four planes of ions are considered, two above and two symmetrical below the $\mathrm{K}^{+}$plane, the closest two with oxygen and the other two with silicon ions. The path for the central $\mathrm{K}^{+}$ion used the in calculation is shown; note that the $\mathrm{O}^{-2}$ ions in the middle of the path are actually at a distance of $1.68 \AA$ above and below. The interaction between the $\mathrm{K}^{+}$ions in the central $X$ axis is not taken into account in constructing the substrate potential as it is taken into account explicitly. The crystal is continued in the $\mathrm{K}^{+}$plane until the convergence is achieved. Distance between the longer marks is $1 \AA$.

mation by a truncated Fourier series:

$$
U_{s}(x)=\sum_{n=0}^{4} U_{n} \cos (2 \pi n x)
$$

with coefficients $U_{n}$ equal to $\{2.4473,-3.3490,1.0997$, $-0.2302,0.0321\}$. The corresponding linear frequency is $\omega_{0}=$ 4.48 or $119 \mathrm{~cm}^{-1}$ in physical units, which is close to $110 \mathrm{~cm}^{-1}$ determined experimentally [47]. Also the potential well of $20 \mathrm{eV}$ is consistent with molecular dynamics simulations [48]. It is represented in Fig. 10 together with the other potentials, such that their relative magnitudes can be compared.

The phonon spectrum becomes an optical one, and the frequency and group velocity are

$$
\begin{aligned}
& \omega^{2}=\omega_{0}^{2}+4 c_{s}^{2} \sin ^{2}(q / 2), \\
& V_{g}=\frac{d \omega}{d q}=\frac{c_{s}^{2} \sin q}{\sqrt{\omega_{0}^{2}+4 c_{s}^{2} \sin ^{2}(q / 2)}} .
\end{aligned}
$$

Note that $c_{s}$ is still the sound speed in the system without substrate. The dimensionless phonon frequencies $\omega$ are in the interval between $\omega_{0}=4.48$ and $\omega_{\max }=5.31$. The group velocity is zero at $q=0$ and $q=\pi$ and reaches its maximum $V_{\mathrm{g}, \max } \simeq 0.4$ in proximity to $q=\pi / 2$ with $\lambda \simeq 4$. These features are observed in the simulations.

\section{A. Qualitative description}

The introduction of the substrate potential does not prevent the existence of supersonic lattice kinks. The lattice kink, also called crowdion, consists of an interstitial atom propagating very fast in the lattice and leaving behind a vacancy. The 


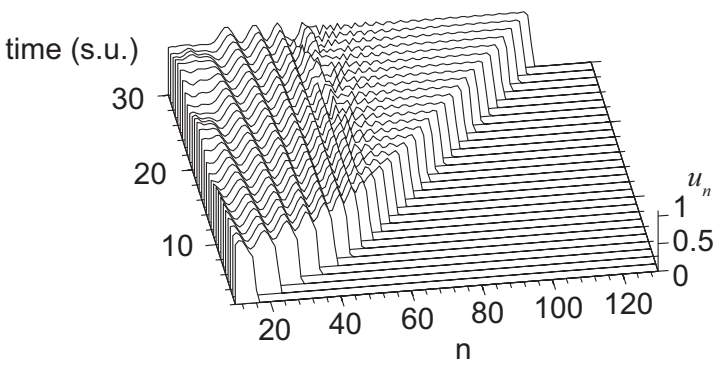

FIG. 12. Representation of the process of dynamical slowing down of the kink. The nonlinear and linear radiation processes can be easily distinguished. Nonlinear waves with large amplitude are first emitted. Later, the phonons with the wavelength close to $\lambda \simeq 4$, which corresponds to maximal group velocity, and with exponentially decaying amplitudes are emitted while the kink approaches the limit velocity. Scaled units for time and distance are $0.2 \mathrm{ps}$ and lattice constant $5.19 \AA$.

specific feature of the kinks found in the present work is that its velocity and energy are fixed by the layer+substrate system, let us denote them as $V_{c}$ and $E_{c}$ ( $c$ for crowdion). If the initial energy is smaller than $E_{c}$, the kinks are rapidly dispersed and disappear, and if it is larger, the excess energy is radiated as the kink slows down to $V_{c}$. The specific values in scaled units are $V_{c}=2.7387 \simeq 2 c_{s}$ and $E_{c}=9.4374$, corresponding to $7.16 \mathrm{~km} / \mathrm{s}$ and $26.2 \mathrm{eV}$, respectively. The lattice kink is supersonic in the two meanings (1) $V_{c}>c_{s}$, where $c_{s}$ is the sound velocity without substrate and (2) $V_{c}$ is much larger than the maximum phonon group speed $V_{\mathrm{c}, \max } \simeq 0.4$ in the system with the substrate, but is not larger than the maximum phase velocity, which is unbounded for $q \rightarrow 0$.

\section{B. Slowing down processes}

The process of slowing down to $V_{c}$ is shown in Figs. 12 and 13, where two well-distinguished phases can be identified.

(a) Nonlinear radiation. For an initial energy $E>E_{c}$, the kink progressively loses energy. The particles immediately

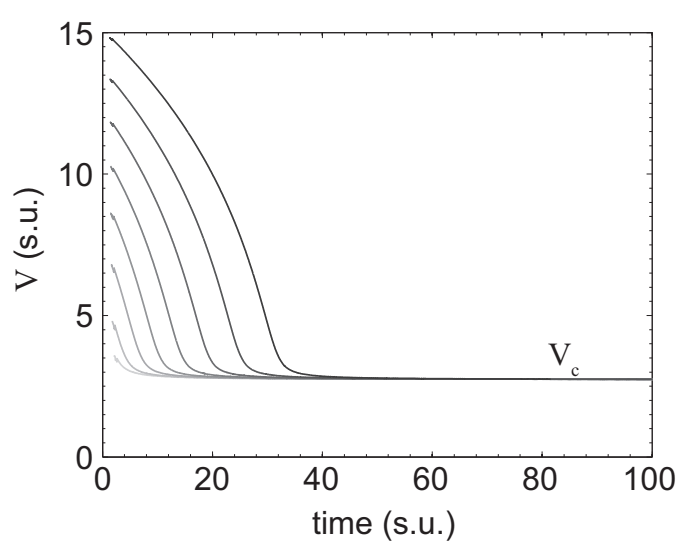

FIG. 13. Velocities of lattice kinks or crowdions versus time in scaled units for different initial conditions. For velocities $V>V_{c}$, the lattice kink slows down until $V=V_{c}$ is reached. For $V<V_{c}$, the kinks are dissipated into low-amplitude phonons. Scaled units are $0.2 \mathrm{ps}$ and $2.6 \mathrm{~km} / \mathrm{s}$. after the kink perturbation are left with enough energy for nonlinear vibrations in the potential well bringing about a nonlinear wave. Their frequencies obtained numerically are above the phonon band with a maximum value of about 6.3 This strong radiative process is shown in Fig. 12 for an initial velocity $V_{0}=7$. This process is very fast, and the loss of energy is almost linear with time.

(b) Linear radiation. As the lattice kink energy approaches $E_{c}$, the amplitude of the tail oscillations and their frequency decrease, and the kink frequency enters the phonon band, radiating low-amplitude phonons [30,31]. The energy decreases exponentially with time towards $E_{c}$. The wave number of the radiated phonons can be deduced from the kink speed as each particle left behind the kink is excited with a delay $\Delta t=1 / V_{c}$ and, therefore, with a phase difference $q=\omega(q) \Delta t=\omega(q) / V_{c}$. As the phonon wave vector is given by $q=\omega(q) / V_{\mathrm{ph}}$, where $V_{\mathrm{ph}}$ is the phase speed of the phonons, $V_{\mathrm{ph}}=V_{c}$. Using the phonon dispersion relation in Eq. (23) it is possible to obtain the phonon wave number and wavelength $\lambda_{\text {ph }}=3.5$, which is the observed one in the simulations. Similar process has been described in Ref. [30] and in references therein for subsonic kinks. However, there is an important difference: The subsonic kinks, described in those works, radiate continuously and eventually stop.

From the comparison of Figs. 8 and 13 with Figs. 10 and 14 , we conclude that the amplitude $A=0.67$ of relative particle displacements of supersonic kink with the unique velocity is determined by the interatomic distance $R$ at the minimum of the total, interatomic plus substrate, potential, $R=1-A \approx 0.33$ in lattice units. This value of the kink amplitude ensures the absence of kink oscillations and correspondingly the absence of phonon radiation into the chain by the supersonic kink. We also want to emphasize that we do not observe a discrete spectrum (a set of possible values) of the velocity of the supersonic kink in our system, even when we start with high initial kink velocity; see Fig. 13. This is in contrast with the prediction of supersonic multisolitons (lattice $N$ solitons) in Frenkel-Kontorova model with nonlinear interparticle coupling in Refs. [31,34,35]. We

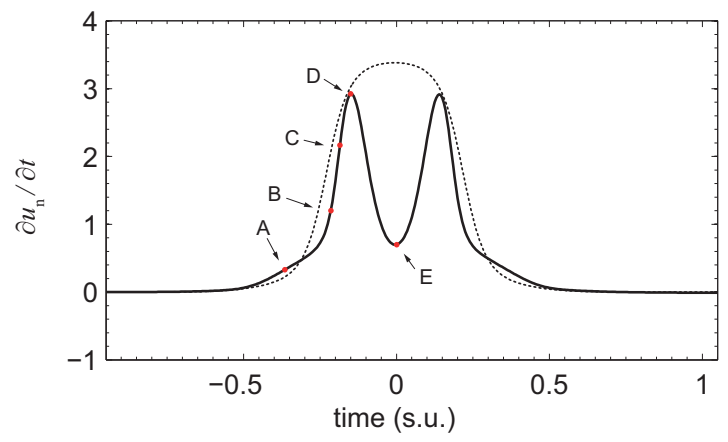

FIG. 14. (Color online) Particle velocity wave form measured at $n=470$ for the supersonic kink with amplitude $A=0.67$ and minimal interatomic distance in lattice units $R=1-A=0.33$ for Coulomb + ZBL potential with (continuous line) and without (dotted line) substrate potential. The instants when the ZBL interaction acts (near A) and the minimum velocity at the top of the potential barrier (E) are easily identified. The configurations at those times can be seen in Fig. 15. Scaled units are $0.2 \mathrm{ps}$ and $2.6 \mathrm{~km} / \mathrm{s}$. 
relate this finding with the extreme discreteness of our kink with the magic wave number $q=2 \pi / 3$, which as shown in Fig. 9 corresponds to only two particles moving at a given time (cf. Ref. [42]) and which does not allow for different matchings with the substrate potential. The uniqueness of the observed supersonic kink velocity we relate also with possible dynamical instability of lattice multisolitons (bound states of supersonic kinks) in the considered system.

\section{Double kink}

The particle velocity $\dot{u}_{n}$ as a function of time for the stable kink is represented in Fig. 14. Due to the extreme discreteness of the kink, it is not practical to represent $\dot{u}_{n}$ an a function of $n$, while the double-kink structure of the found kink in the coordinates, shown in Fig. 15(a), is evident as a function of time.

The extreme discreteness of the kink makes possible the detailed description of the double-kink profile as shown in Fig. 15(c). The particle $n$, represented by a white circle, which is initially at rest at the bottom of a potential well, experiences two sequential collisions: one when it was hit by the particle $n-1$ and is accelerated afterwards, and a second one when it hits the particle $n+1$ and is decelerated, attaining almost zero velocity at the bottom of the potential well (the particle velocity is different from zero during the radiation process). Between the two collisions, the particle finds the substrate potential barrier between the sites and experiences a decrease in velocity while going uphill, which is followed by an acceleration while going downhill.

Note that the states $\mathrm{A}$ and $\mathrm{E}$ in the Fig. 15 have exactly the same energy, but the particle $n$ has moved only half a lattice site. This is the first kink of the double-kink wave form shown in Fig. 15(a). The evolution of the particle $n$, going downhill and hitting the $n+1$ particle until it stops, forms the second kink in the double-kink structure.

This process can also be seen in terms of the kink energy in Fig. 15(b). Two identical oscillations of the particle's potential energy $E_{p}$ happen while the kink travels one lattice site. There is a local maximum at point $C$, corresponding to the minimum distance between particles with the short-range ZBL interaction. The horizontal dashed-dotted lines indicate the energies for the equilibrium interstitial configurations, with two particles inside a potential well (stable), or one particle at the top of the potential barrier (unstable), with the energy difference corresponding to the Peierls-Nabarro (PN) barrier. The potential energy is always above the stable interstitial energy as the lattice has no time to relax, bringing about an adiabatic PN barrier. The kink always has finite kinetic energy, with the minimum reached in the configuration $A$.

\section{SUMMARY}

In this paper we have developed a comprehensive dynamical model of the localized lattice excitations in the lowdimensional system using realistic potentials corresponding to a row of ions in the silicate layer of the mica muscovite crystal.

Our objective was to determine what kind of propagating nonlinear localized excitations can exist in a layered crystal with realistic parameters and with what characteristics. The choice of the parameters of the mica muscovite is motivated by the fact that many of the dark tracks that appear in sheets of this material are consistent with the in-layer propagation
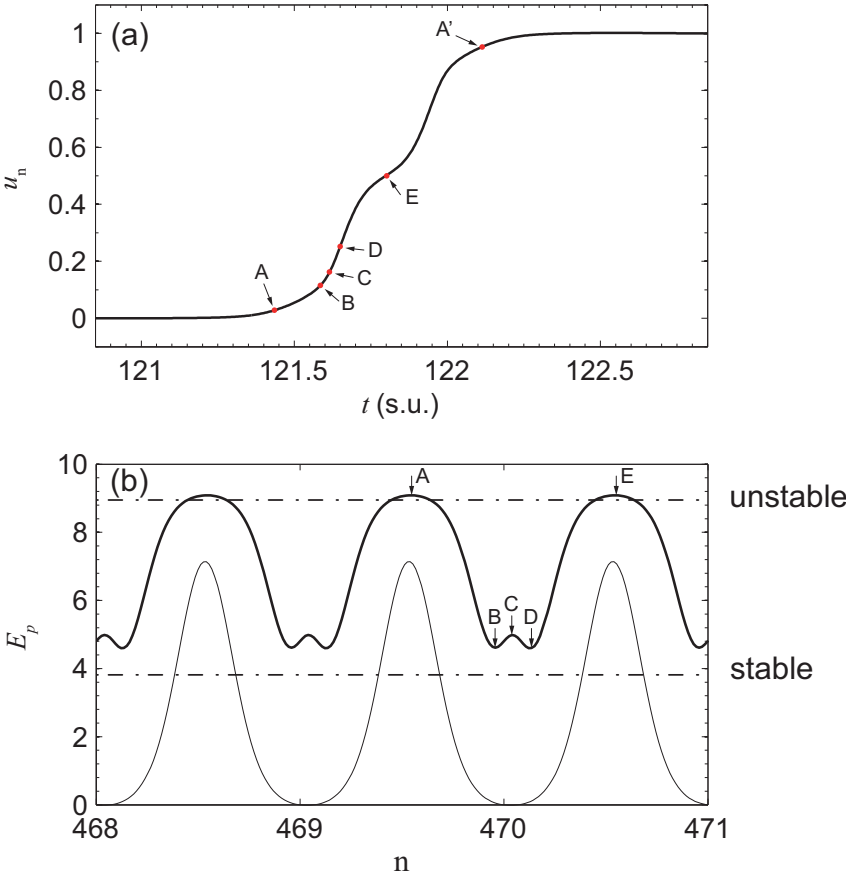

(c)

A

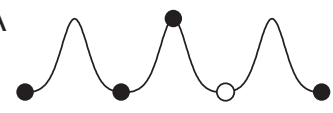

B

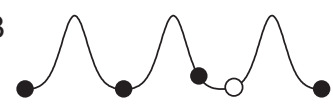

C

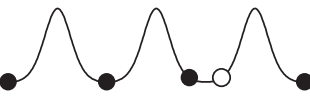

$\mathrm{D}$

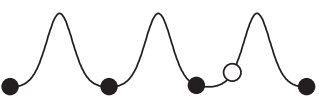

E

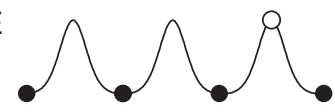

FIG. 15. (Color online) (a) Particle displacement wave form for the established lattice kink. (b) Potential energy seen by the kink during propagation (thick line) and periodic substrate potential (thin line), horizontal lines are the energies corresponding to the equilibrium stable (C) and unstable (A) interstitials. (c) Particle configurations corresponding to the points $A-E$, shown in (a) and (b). Scaled units are lattice unit $5.19 \AA$ A for distances, $0.2 \mathrm{ps}$ for time, and $2.77 \mathrm{eV}$ for energies. 
of localized vibrational excitations along the close-packed lattice lines of ions, and an experiment has demonstrated that localized energetic excitations can travel along the closepacked lattice directions, being able to eject an atom at the opposite surface.

The modeling of the system has followed the process of increasing complexity for better understanding which effect is responsible for which characteristic of the model. In the starting model used in preliminary publications [21,22], only $\mathrm{K}^{+}$ions with nearest-neighbor Coulomb repulsion were taken into account, for which we have found that very fast supersonic kinks can propagate. They are extremely localized, with only two particles or, for higher energies, only a single particle in motion at the same time.

In the present paper we have performed analytical calculations of the displacement patterns and energies of the supersonic kinks within the proposed dynamical model and compare them with numerical simulations. We have also shown that the introduction of the interaction with several nonnearest neighbors does not produce significative changes as long as the sound speed in each system is taken as a reference. The extremely short minimal interatomic distances in the kink has motivated us to introduce more realistic short-range Ziegler-Biersack-Littmark repulsive potential. In the improved dynamical model, kinks propagate equally well and with as high energy as desired, and with realistic minimal interatomic distances.

The next step was the introduction of a periodic substrate potential using empirical potentials and the geometry of the layered crystal. Supersonic kinks continue to propagate without losing energy but with several important properties: (a) The main one is that the system selects only single velocity and single energy of the kink; (b) the energy of the lattice kink is larger than the one which is needed for atom ejection at the surface and is smaller than the one of the proposed sources of energy, the recoil of a $\mathrm{K}^{+}$ion due to $\beta$ emission; and (c) the found kinks can be described as double kinks or bisolitons depending on the dynamical variable chosen. The unique average velocity of the supersonic kink on the periodic substrate potential we relate with the kink amplitude of the relative particle displacements, which is determined by the interatomic distance corresponding to the minimum of the total, interparticle plus substrate, lattice potential.

The found kinks are ultra discrete and can be described with the magic wave number $q=2 \pi / 3 a$, which was previously revealed in the nonlinear sinusoidal waves and supersonic kinks in the Fermi-Pasta-Ulam lattice $[41,42]$. The extreme discreteness of the supersonic kinks observed in our work, with basically two particles moving at the same time, allows for the detailed interpretation of their double-kink structure, which is not possible for the multikinks without an account for the lattice discreteness. The double-kink structure is produced by the matching of the two sequential collisions experienced by a particle with the process of going over the substrate potential barrier between neighboring sites.

The computed energy of the supersonic kinks found in the realistic lattice potential considered is approximately $26 \mathrm{eV}$. Such energy can be provided by the recoil of isotopes of potassium after radioactive decay and it is larger than the sputtering energy. This value of the characteristic energy of the supersonic kinks found allows us to assume that the tracks found in mica muscovite crystals can be related with the propagating lattice kinks.

\section{ACKNOWLEDGMENTS}

J.F.R.A., V.S.M., and L.M.G.R. acknowledge financial support from the projects FIS2008-04848, FIS2011-29731C02-02, and MTM2012-36740-C02-02 from Ministerio de Ciencia e Innovación (MICINN). All authors acknowledge Prof. F. M. Russell for ongoing discussions.
[1] S. A. Durrani, A success story of the 20th century, Rad. Meas 34, 5 (2001).

[2] S. A. Durrani, Nuclear tracks today: Strengths, weaknesses, challenges, Rad. Meas 43, S26 (2008).

[3] E. C. H. Silk and R. S. Barnes, Examination of fission fragment tracks with an electron microscope, Philos. Mag. 4, 970 (1959).

[4] P. B. Price and R. M. Walker, Observation of fossil particle tracks in natural micas, Nature (London) 196, 732 (1962).

[5] R. Fleischer, Tracks to Innovation. Nuclear Tracks in Science and Technology (Springer, New York, 2011).

[6] D. P. Snowden-Ifft, E. S. Freeman, and P. B. Price, Limits on dark-matter using ancient mica, Phys. Rev. Lett. 74, 4133 (1995).

[7] M. D. Alba, A. I. Becerro, M. A. Castro, and A. C. Perdigón, Hydrothermal reactivity of Lu-saturated smectites: Part I. A long-range order study, Am. Mineral. 86, 115 (2001).

[8] Z. L. Hong, H. Yoshida, Y. Ikuhara, T. Sakuma, T. Nishimura, and M. Mitomo, The effect of additive on sintering behavior and strength retention in silicon nitride with RE-disilicate, J. Eur. Ceram. Soc. 22, 527 (2002).

[9] J. F. R. Archilla, J. Cuevas, M. D. Alba, M. Naranjo, and J. M. Trillo, Discrete breathers for understanding reconstructive mineral processes at low temperatures, J. Phys. Chem. B 110, 24112 (2006)

[10] V. I. Dubinko, P. A. Selyshchev, and J. F. R. Archilla, Reactionrate theory with account of the crystal anharmonicity, Phys. Rev. E 83, 041124 (2011).

[11] F. M. Russell, Tracks in mica caused by electron showers, Nature (London) 216, 907 (1967).

[12] F. M. Russell, Duration of sensitive period for track recording in mica, Nature (London) 217, 51 (1967).

[13] F. M. Russell, Positive charge transport in layered crystalline solids, Phys. Lett. A 130, 489 (1988).

[14] F. Russell, Identification and selection criteria for charged lepton tracks in mica, Nucl. Tracks. Rad. Meas. 15, 41 (1988).

[15] J. A. Cameron and B. Singh, Nuclear data sheets for $A=40$, Nucl. Data Sheets 102, 293 (2004).

[16] X. Mougeot and R. G. Helmer, http://www . nucleide.org .

[17] D. Schlößer, K. Kroneberger, M. Schosnig, F. M. Russell, and K. O. Groeneveld, Search for solitons in solids, Rad. Meas 23, 209 (1994).

[18] F. M. Russell and J. C. Eilbeck, Evidence for moving breathers in a layered crystal insulator at 300K, Europhys. Lett. 78, 10004 (2007). 
[19] Y. Kudriavtsev, A. Villegas, A. Godines, and R. Asomoza, Calculation of the surface binding energy for ion sputtered particles, Appl. Surf. Sci. 239, 273 (2005).

[20] Q. Dou, J. Cuevas, J. C. Eilbeck, and F. M. Russell, Breathers and kinks in a simulated crystal experiment, Discret. Contin. Dyn. Syst. S 4, 1107 (2011).

[21] J. F. R. Archilla, Yu. A. Kosevich, N. Jiménez, V. J. SánchezMorcillo, and L. M. García-Raffi, Moving excitations in cation lattices, Ukr. J. Phys. 58, 646 (2013).

[22] J. F. R. Archilla, Yu. A. Kosevich, N. Jiménez, V. J. SánchezMorcillo, and L. M. García-Raffi, Supersonic Kinks in Coulomb Lattices, in Localized Excitations in Nonlinear Complex Systems, edited by R. Carretero et al. (Springer, New York, 2014), pp. 317-331.

[23] J. Biersack, J. P. Ziegler, and M. D. Ziegler, SRIM: The Stopping and Range of Ions in Matter (J. P. Ziegler, Chester, Maryland, 2008).

[24] A. V. Savin and Yu. A. Kosevich, Thermal conductivity of molecular chains with asymmetric potentials of pair interactions, Phys. Rev. E 89, 032102 (2014).

[25] J. B. Gibson, A. N. Goland, M. Milgram, and G. H. Vineyard, Dynamics of radiation damage, Phys. Rev. 120, 1229 (1960).

[26] A. Meftah, F. Brisard, J. M. Costantini, E. Dooryhee, M. Hage-Ali, M. Hervieu, J. P. Stoquert, F. Studer, and M. Toulemonde, Track formation in $\mathrm{SiO}_{2}$ quartz and the thermal-spike mechanism, Phys. Rev. B 49, 12457 (1994).

[27] C. Trautmann, S. Klaumünzer, and H. Trinkaus, Effect of stress on track formation in amorphous iron boron alloy: Ion tracks as elastic inclusions, Phys. Rev. Lett. 85, 3648 (2000).

[28] Ya. Frenkel and T. Kontorova, On the theory of plastic deformation and twinning, Phys. Z. Sowjetunion 13, 1 (1938).

[29] P. M. Chaikin and T. C. Lubensky, Principles of Condensed Matter Physics (Cambridge University Press, Cambridge, 1995).

[30] O. M. Braun and Y. S. Kivshar, Nonlinear dynamics of the Frenkel-Kontorova model, Phys. Rep. 306, 1 (1998).

[31] O. M. Braun and Y. S. Kivshar, The Frenkel-Kontorova Model: Concepts, Methods, and Applications (Springer, Berlin, 2004).

[32] A. M. Kosevich and A. S. Kovalev, The supersonic motion of a crowdion. The one dimensional model with nonlinear interaction between the nearest neighbors, Solid State Commun. 12, 763 (1973).
[33] A. Milchev, Breakup threshold of solitons in systems with nonconvex interactions, Phys. Rev. B 42, 6727 (1990).

[34] A. V. Savin, Supersonic regimes of motion of a topological soliton, Sov. Phys. JETP 81, 608 (1995).

[35] Y. Zolotaryuk, J. C. Eilbeck, and A. V. Savin, Bound states of lattice solitons and their bifurcations, Physica D 108, 81 (1997).

[36] Y. Ni, Yu. A. Kosevich, S. Xiong, Y. Chalopin, and S. Volz, Substrate-induced cross-plane thermal propagative modes in few-layer graphene, Phys. Rev. B 89, 205413 (2014).

[37] Y. Pomeau, Front motion, metastability and subcritical bifurcations in hydrodynamics, Physica D 23, 3 (1986).

[38] M. C. Cross and P. C. Hohenberg, Pattern formation outside of equilibrium, Rev. Mod. Phys. 65, 851 (1993).

[39] M. G. Clerc, R. G. Elías, and R. G. Rojas, Continuous description of lattice discreteness effects in front propagation, Philos. Trans. R. Soc. A 369, 412 (2011).

[40] P. C. Matthews and H. Susanto, Variational approximations to homoclinic snaking in continuous and discrete systems, Phys. Rev. E 84, 066207 (2011).

[41] Yu. A. Kosevich, Nonlinear sinusoidal waves and their superposition in anharmonic lattices, Phys. Rev. Lett. 71, 2058 (1993).

[42] Yu. A. Kosevich, R. Khomeriki, and S. Ruffo, Supersonic discrete kink-solitons and sinusoidal patterns with magic wave number in anharmonic lattices, Europhys. Lett. 66, 21 (2004).

[43] P. Poggi and S. Ruffo, Exact solutions in the FPU oscillator chain, Physica D 103, 251 (1997); Yu. A. Kosevich and S. Lepri, Modulational instability and energy localization in anharmonic lattices at finite energy density, Phys. Rev. B 61, 299 (2000).

[44] G. Friesecke and K. Matthies, Atomic-scale localization of highenergy solitary waves on lattices, Physica D 171, 211 (2002).

[45] M. Molerón, A. Leonard, and C. Daraio, Solitary waves in a chain of repelling magnets, J. Appl. Phys. 115, 184901 (2014).

[46] O. Gedeon, J. Macháek, and M. Liška, Static energy hypersurface mapping of potassium cations in potassium silicate glasses, Phys. Chem. Glasses 43, 241 (2002).

[47] M. Diaz, V. C. Farmer, and R. Prost, Characterization and assignment of far infrared absorption bands of $\mathrm{K}^{+}$in muscovite, Clays Clay Miner. 48, 433 (2000).

[48] D. R. Collins and C. R. A. Catlow, Computer simulation of structure and cohesive properties of micas, Am. Mineral. 77, 1172 (1992). 